\title{
Die Verfahrensautonomie der Mitgliedstaaten der Europäischen Union
}

CHRISTOPH KRÖNKE

Die Verfahrensautonomie der Mitgliedstaaten der Europäischen Union

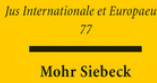

2013. XXII, 383 Seiten. JusIntEu 77

ISBN 978-3-16-152642-8

DOI 10.1628/978-3-16-152642-8

eBook PDF $89,00 €$

ISBN 978-3-16-152641-1

Leinen $89,00 €$
Die Verfahrensautonomie der Mitgliedstaaten der Europäischen Union zählt zu den umstrittensten Leitfragen des Europäischen Verwaltungsrechts. Christoph Krönke rekonstruiert die mitgliedstaatliche Verfahrensautonomie auf der Grundlage eines prinzipientheoretischen Ansatzes im Spannungsverhältnis von nationaler Eigenverantwortung und europäischer Integration. Dabei erweisen sich das Äquivalenz- und das Effektivitätsprinzip als spezifischer Ausdruck des Grundsatzes der Verhältnismäßigkeit. Dies führt zu einer umfassenden Kritik der Rechtsprechung des Europäischen Gerichtshofs im Hinblick auf die Bestandskraft von Verwaltungsakten und die Rechtskraft von Gerichtsentscheidungen.

Christoph Krönke Geboren 1983; 2003-09 Studium der Rechtswissenschaft in Heidelberg und München; 2013 Promotion (LMU München); Rechtsreferendariat im OLG-Bezirk München; Wissenschaftlicher Mitarbeiter; seit 2017 Akademischer Rat a.Z. am Lehrstuhl für Öffentliches Recht, Wirtschaftsverwaltungsrecht, Umwelt- und Sozialrecht der LMU München; 2020 Habilitation (LMU München); seit September 2020 Universitätsprofessor an der Wirtschaftsuniversität Wien.

Jetzt bestellen:

https://mohrsiebeck.com/buch/die-verfahrensautonomie-der-mitgliedstaaten-der-europaeischen-union-9783161526428? no_cache=1

order@mohrsiebeck.com

Telefon: +49 (0)7071-923-17

Telefax: $+49(0) 7071-51104$ 